\begin{tabular}{ccc}
\hline \multicolumn{2}{c}{ A N N A LES } \\
UNIVERSITATIS M A R I A E C URIE-SKŁ ODOWSK A \\
LOL. XXXVI & SECTIOFF & $1-2018$ \\
\hline
\end{tabular}

\title{
LIHUA JIN
}

ORCID ID: https://orcid.org/0000-0001-5015-1489

Université Paris-Sorbonne

\section{L'étude contrastive dans la perspective de la didactique de l'écrit en L2: les trois axes de la rhétorique}

Badania kontrastywne w perspektywie dydaktyki pisania w L2:

trzy osie retoryki

Contrastive study in the perspective of didactics of writing in L2: The three axes of rhetoric

La distinction établie entre la langue et la parole, qui a marqué il y a un siècle le commencement de la linguistique moderne, a élargi considérablement le champ d'études de la linguistique. L'intégration de la dimension discursive en son sein a donné lieu à de nouvelles approches méthodologiques qui permettent des descriptions toujours plus fines de la structure linguistique.

Cette nouvelle perception de la langue se caractérise par l'accent mis sur l'aspect pragmatico-social de la langue, comparé à l'aspect grammatico-syntaxique de la linguistique traditionnelle. Cette tendance est confirmée plus particulièrement par nombre de recherches portant sur les données des interactions verbales produites en situation authentique dans la didactique des langues étrangères, et ce, attribuant une place plus importante à l'oral qu'à l'écrit.

C'est pour ainsi dire que les enseignants se trouvent souvent démunis de méthodes adaptées pour évaluer et améliorer la qualité des écrits des scripteurs d'une langue étrangère et s'y aventurent peu à cause de la complexité multiple qui s'impose au-delà du niveau phrastique. Pour un enseignement plus efficace de l'écrit de L2, beaucoup de facteurs textuels doivent être pris en compte et ont encore besoin d'être décrits de façon systémique. 
Cet article propose une définition du texte comme objet d'étude et présente la rhétorique contrastive comme une solution méthodologique dans le domaine de la didactique de l'écrit de L2. De même, cet article vise à établir des liens entre des dans toutes sortes de phénomènes textuels traités en rhétorique contrastive, en se référant à la perspective de l'analyse textuelle des discours J.-M. Adam.

\section{LE TEXTE PRODUIT PAR LES APPRENANTS DE L2}

\subsection{La didactique de l'écrit de L2}

La rhétorique contrastive est née en 1966 dans le domaine de l'enseignement de l'anglais langue seconde pour un besoin didactique de l'écrit.

Chaque langue est caractérisée par un ensemble de conventions rhétoriques qui lui sont propres. Ces conventions influencent la façon de penser et d'écrire des dépositaires de cette culture et par conséquent interfèrent avec leur écriture en langue étrangère. (Kaplan, 1966; Grabe et Kaplan, $1989 ; 2002$; cité in Bi, 2016)

Ainsi, la rhétorique contrastive est à la fois «un domaine de recherche en acquisition d'une langue seconde qui identifie les problèmes de production rencontrés par des scripteurs en langue seconde» et propose « des explications en faisant référence aux stratégies rhétoriques de la langue maternelle» (Connor, 1996, cité in $\mathrm{Bi}, 2016)$.

Avant tout, les recherches contrastives des textes produits par les apprenants de L2 nécessitent une définition rigoureuse du texte comme objet d'étude. E. Benveniste propose une vision englobant qui ne voit l'existence de la langue que dans la parole. Et cette dernière intègre dans l'ensemble de la structure linguistique une série des phénomènes discursifs comme la relation inférentielle, la situation d'énonciation, etc. Benveniste rappelle que «c'est dans le discours, actualisé en phrases, que la langue se forme et se configure. Là commence le langage» (Benveniste, 1966: 131). C'est ainsi que la dimension discursive devient partie intégrante de la structure linguistique et qu'est née cette linguistique marquée par l'énonciation et centrée sur la question du discours.

\subsection{Le texte comme objet d'étude linguistique}

Benveniste distingue trois sortes de linguistique. La première est «sémiotique», qui caractérise la langue par un système de signes dont l'unité est le signe. C'est un champ d'étude dont l'objet est proche de celui de langue de Saussure. La 
deuxième branche est celle de la linguistique du discours ou «sémantique», qui a pour objet l'acte même de produire les énoncés du discours dont l'unité est la phrase comme étant unité de sens. Enfin, la dernière branche est celle de l'analyse «translinguistique des textes et des œuvres». Ce sera une sémiologie de «deuxième génération », dont les instruments et la méthode pourront aussi concourir au développement des autres branches de la sémiologie générale (Benveniste, 1974; cité in Adam, 2005).

Ces trois champs linguistiques identifiés par Benveniste sont présentés sous forme de schéma par Adam (2005) à la fois discontinu (opposant deux ensembles) et continu (la linguistique de l'énonciation assurant la transition entre les deux domaines auxquels elle appartient).

Analyse translinguistique

\begin{tabular}{|l|l|l|}
\cline { 2 - 3 } $\begin{array}{l}\text { Signifiance du signe } \\
\text { "sémiotique " }\end{array}$ & $\begin{array}{l}\text { Signifiance du } \\
\text { discours } \\
\text { "sémantique \# }\end{array}$ & $\begin{array}{l}\text { Métasignifiance } \\
\text { des textes } \\
\text { et } \\
\text { des œuvres } \\
\text { «métasémantique » }\end{array}$ \\
\hline
\end{tabular}

Analyse intralinguistique

Schéma 1. Trois champs linguistiques - cette représentation a été simplifiée et modifiée du schéma d'origine d'Adam (2005: 15)

Cette représentation schématique permet de bien appréhender notre objet d'analyse - le texte produit par les apprenants dans un contexte particulier de la production -, en le situant sur la lignée de l'analyse translinguistique dont le fondement théorique se trouve sur la sémantique de l'énonciation.

\subsection{La distinction entre le texte et le discours}

La distinction traditionnellement acceptée en linguistique entre le texte et le discours est la présence d'un contexte de production. Le discours exige notamment à la fois l'existence des propriétés textuelles et celle de la situation de communication particulière. Et le texte, quant à lui, il est en quelque sorte l'objet formel, des données verbales, soustrait de l'ensemble du discours prononcé. Mais cette distinction est devenue contraignante lors qu'on constate que la production du texte n'est pas soumise strictement au seul système fonctionnel de la langue. Pour Hoey (1991) le texte «se réfère non seulement à un document oral ou écrit qui peut être analysé linguistiquement, mais également à un niveau linguistique situé entre la syntaxe et l'interaction» (Hoey, 1991; cité in Carter-Thomas, 2000). 
On peut constater que le rapprochement entre le texte et le discours va de soi, au fil de l'évolution des réflexions qui au tout début les distinguaient avec la présence du contexte de production. Adam, dans son travail, cite A. Ali Bouacha qui a bien posé le rapport entre le discours et le texte, le discours comme «objet empirique renvoyant à du texte» :

D'un côté on met en relation du texte dans son appréhension immédiate avec une activité discursive à laquelle il renvoie ou plus précisément à laquelle on le fait renvoyer, et de l'autre, on considère ce texte comme un ensemble de données linguistiques brutes qu'il faut ensuite traiter en données discursives. Celles-ci sont nécessairement filtrées par des propriétés linguistiques à partir desquelles il est possible de décrire des propriétés discursives. (Ali Bouacha, 1993; cité in Adam, 2005)

Comme ceci explique qu'en pratique la séparation rigoureuse entre le texte et le discours est peu réalisable et contraignante vue la complémentarité des deux objets linguistiques. Toutes pratiques discursives humaines qu'elles soient réalisées sous forme d'oral ou d'écrit possèdent nécessairement des «données linguistiques brutes » et en même temps s'inscrivent à un contexte particulier de production renvoyant à une activité singulière discursive.

Postulant, à la fois, une séparation et une complémentarité des tâches et des objets de la linguistique textuelle et de l'analyse de discours, Adam (2005) définit la linguistique textuelle comme un sous-domaine du champ plus vaste de l'analyse des pratiques discursives et propose de l'inscrire à ce double titre.

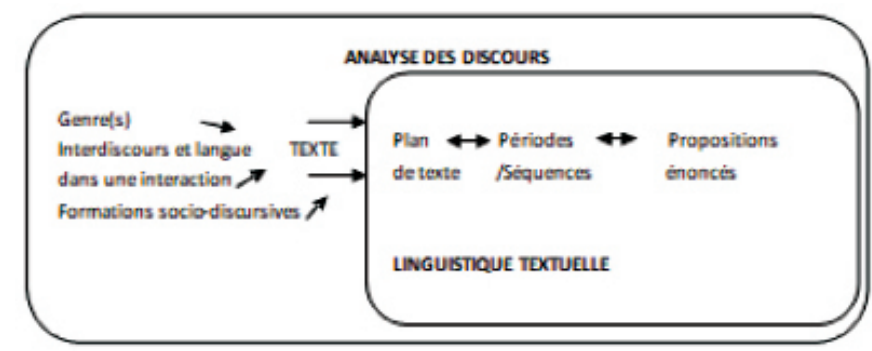

Schéma 2. L'analyse des pratiques discursives - cette représentation est la version reproduite et simplifiée du schéma d'origine de J.-M. Adam (2005: 19)

La linguistique textuelle a pour rôle, au sein de l'analyse de discours, de théoriser et de décrire les agencements d'énoncés élémentaires au sein de l'unité de haute complexité que constitue un texte. Chaque texte se présente comme un énoncé complet, mais non isolé, et comme le résultat toujours singulier d'un acte d'énonciation. C'est, par excellence, l'unité de l'interaction humaine. (Adam, 2005: 28-29) 
La complexité de la nature du texte nous exige à prendre en compte à la fois des procédés formels de connexion des unités du texte et des facteurs de situation, d'interdiscours, d'intersubjectivité, de formation socio-discursive, etc.

\section{LA LINGUISTIQUE TEXTUELLE COMME UNE THÉORIE DE FONDEMENT DE LA RHÉTORIQUE CONTRASTIVE}

Partant de la nature du corpus étudié en rhétorique contrastive, nous considérons que le rapprochement entre la rhétorique contrastive et la linguistique textuelle des discours est un choix théorique d'évidence.

\subsection{Les objets d'étude en rhétorique contrastive}

Dans la rhétorique contrastive, on analysait toutes sortes de caractéristiques du texte écrit comme le phénomène d'anaphore, la reprise lexicale, la nominalisation, les liens cohésifs entre des propositions-énoncés, les connecteurs, les relations thème - rhème, des structures de la macrostructure et la superstructure (Van Dijk, 1980, 1985), la description illocutoire et du rôle interactif de la structure du texte argumentatif, basée sur la théorie des actes de langage (Tirkonnen-Condit, 1985), les relations cohésives de Halliday et Hasan (1976) ainsi que bien d'autres recherches ont contribué à l'épanouissement du domaine. Néanmoins, la plupart des études de la rhétorique contrastive datant de cette époque portent sur les comparaisons entre l'anglais et d'autres langues lointaines.

Les recherches francophones sur le texte et le discours sont centrées sur la question du genre et de la typologie du texte comme celles d'Adam (1985), sur la cohésion et la cohérence textuelle de Charolles $(1982,1988)$ sur la grammaire de texte de Combettes (1983) et sur la théorie de l'argumentation d'Anscombre (1983, 1984, 1995) qui étudie les enchaînements des énoncés. Il paraît que les résultats de ces travaux français n'ont pas donné grande inspiration à la rhétorique contrastive et ont été encore moins appliquée dans la didactique du français langue étrangère.

\subsection{La catégorisation des phénomènes textuels traités}

D’ailleurs, on constate que des caractéristiques rhétoriques recherchées dans des études effectuées sont très variées sans avoir établi de corrélations entre des phénomènes traités au sein du domaine de la rhétorique contrastive. De façon très primaire, on peut les classer en deux catégories : les études traitant des caractéristiques locales et des caractéristiques globales. 


\subsubsection{Les caractéristiques locales}

Au début des années 80 et 90 les recherches sur le corpus des textes écrits se penchaient sur la comparaison des traits syntaxico-grammaticaux qui apparaissent localement dans le texte, par exemple comparer la longueur des phrases produites par les apprenants de l'anglais L2 et celles produites par les scripteurs anglophones. On y recensait le nombre de conjonctions de coordination et de subordination, le nombre de connecteurs, le nombre de phrases simples, complexe: proposition conjonctive, proposition subordonnée, etc. Mais ce genre d'étude n'a pas pu donner d'explication convaincante expliquant l'origine de ces écarts, voire plus loin, aboutir à une solution pédagogique dans la didactique de l'écrit en L2.

\subsubsection{Les caractéristiques globales}

N'ayant pas trouvé de réponse satisfaisante au niveau de «micro-structure», certains rhétoriciens se sont aventurés sur le terrain des caractéristiques globales du texte, notamment celles qui décident la cohérence et la superstructure qui réfère à la façon dont un texte s'organise à l'intérieur d'un genre textuel particulier comme des travaux de Connor et Kaplan publiés en 1987 et de Purves (en 1988). Mais ce genre d'études n'a pu éclairer l'hypothèse sur l'organisation textuelle telle que l'organisation des paragraphes linéaire ou digressive, les procédés de raisonnement déductif ou inductif, des traits rhétoriques souvent mis à l'opposé pour catégoriser la rhétorique textuelle selon la culture d'origine des scripteurs.

\subsection{La perspective contrastive}

On peut développer plusieurs formes de comparaison suivant des paramètres de comparaison différents. Quant au paramètre de la variété de langue, il peut y avoir deux configurations possibles:

a) La comparaison des textes écrits dans des langues différentes - des textes écrits en L1 d'origine, qui sont ensuite traduits en L2 pour être comparés avec des textes écrits en L2 d'origine. Cette configuration consiste à contraster la traduction des textes produits en différentes L1 avec des textes originellement produits en L2, en présumant que l'on peut expliquer l'origine de certaines maladresses dans la production écrite des apprenants se trouve dans les conventions rhétoriques de L1. Cette forme de comparaison permet d'accentuer des traits rhétoriques distinctifs entre des langues comparées.

b) La comparaison des textes originellement produits en une langue commune - textes produits par les apprenants de L2 en L2 vs textes produits par les scripteurs natifs en leur langue maternelle (L2 des apprenants). La particularité de cette configuration est que l'on peut observer des maladresses textuelles issues 
des difficultés particulières quand les deux conventions rhétoriques s'interposent surtout à un moment précis au cours de l'acquisition de L2. Cette forme de comparaison permet aussi de vérifier si certaines caractéristiques rhétoriques sont communément présentes chez les individus partageant la même langue maternelle.

En outre, la comparaison peut aussi être portée sur la compétence rédactionnelle acquise des scripteurs. On peut les séparer en deux catégories: les scripteurs experts et les scripteurs novices. Ce paramètre est souvent adopté en situation d'acquisition de L1.

\section{LES TROIS AXES DE LA RHÉTORIQUE CONTRASTIVE}

Depuis les premiers travaux de Kaplan et tant d'autres études, les caractéristiques rhétoriques se trouvent éparpillées au sein de la rhétorique si bien que chaque étude a sa liste prioritaire à traiter. Et chacune prétend des solutions pédagogiques du point de vue de la rhétorique, et ce sans situer ses objets d'étude ou préciser la portée de l'application du résultat d'analyse dans la pratique de l'enseignement. Bien que la rhétorique soit difficile d'être conceptualisée, nous essayons, comme a fait A. Purves (1986), de lui attribuer trois grands axes constitutifs qui à la fois classifient et mettent en lien des caractéristiques particulières comparées comme étant objets d'études de la rhétorique contrastive. Ces trois axes sont «argumentation, organisation et style» respectivement.

Although rhetoric is a notoriously difficult concept to define, three constituent elements have generally been recognized by researchers : «organization, style, and argumentation». (Purves, 1986: 50)

Par la suite, nous essayons d'illustrer la relation entre ces axes proposés en les mettant en correspondance avec le schéma d'Adam (2005: 19) et en citant des études récentes du domaine partageant la même perspective d'analyse.

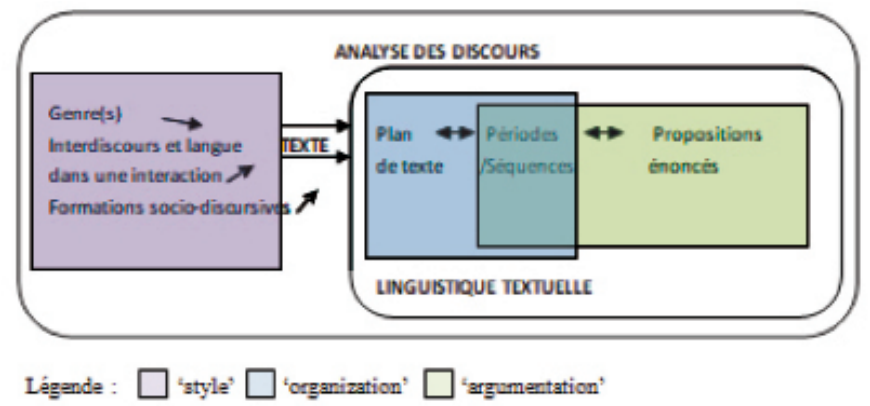

Schéma 3. Les trois axes de la rhétorique correspondant au schéma des objets de la linguistique textuelle des discours 


\subsection{L'axe de l'argumentation}

Dans l'axe présent, nous regroupons les études sur les enchaînements des propositions-énoncés dont un sert d'argument et l'autre de conclusion. Il s'agit de tous procédés d'argumentation, qu'ils soient sémantiques ou discursifs, qui sont mis en place pour créer la texture d'un texte. Sous cette rubrique, on peut inscrire des marques explicites linguistiques ayant des fonctions du discours identifiables à la cohésion.

Par exemple, Hallyday et Hasan (1976) font certains procédés ou marqueurs - anaphore, conjonction, etc. - des conditions de la cohérence textuelle. On peut attribuer à ce genre d'étude un caractère d'étude de signalisation.

Pour un exemple récent du français, on peut citer Saneikashani (2012) qui arrive à la même conclusion que Halliday et Hasan que certains types de relations cohésives sont réalisés par la grammaire telle que la référence, la substitution et l'ellipse. Et d'autres sont réalisés par le lexique. Dans l'étude de Saneikashani (2012) les relations conjonctives sont considérées comme l'exemple parfait qui relève les relations cohésives du texte. De ce fait, en recourant principalement aux relations conjonctives, elle vise à démontrer l'acquisition de l'emploi et de la variété des indices de cohésion dans l'apprentissage de L2.

Le rapport argumentatif entre les propositions-énoncés qui se succèdent peut être exprimé et signalé par une variété d'expressions (conjonctions, adverbes, syntagmes prépositionnels), que nous regroupons sous l'appellation large de connecteurs. Les études contrastives portant sur l'utilisation des connecteurs peuvent être servies comme une sorte d'étude sur la cohésion textuelle.

La progression thématique (Combettes, 1983) est une autre façon par laquelle on étudie l'agencement des phrases en une suite, qui tienne compte à la fois de la hiérarchie et du degré de nouveauté des informations à l'intérieur d'un texte. On peut associer à l'axe d'«argumentation» le niveau «micro-structure» où la relation de connexité est explicitée par les signalisations linguistiques que l'on peut isoler en unité grammaticale ou lexicale. Alors que dans l'axe organisationnel nous passons au niveau supérieur de la «macro-structure».

\subsection{L'axe de l'organisation}

Dans cet axe, on se situe entre le niveau «macro-structure» (les agencements de paquets de propositions sémantiquement regroupés) et ce que Van Dijk (1980) appelle «superstructure», les schémas de texte régulés par les organisations conventionnelles. Van Dijk a proposé d'appeler «superstructures» : 
A la différence des macrostructures, elles ne déterminent pas un «contenu» global, mais plutôt la «forme» globale d'un discours [...]. Les macro-propositions, au moins celles d'un niveau assez élevé, seront organisées par les catégories schématiques de la superstructure, par exemple, le schéma narratif. (cité in Adam, 2005: 138)

À la différence d'une approche qui est centrée sur les phénomènes de cohésion formelle, une analyse de la structuration thématique que propose Carter-Thomas (2000) permet de lier une étude sur les phénomènes formels de liens intra- et interphrastiques à une appréciation plus individualisée (subjective) de la cohérence textuelle.

Carter-Thomas a choisi une analyse qui privilégie l'organisation thématique du texte, parce qu'il croit que la structure thématique permettra d'analyser efficacement certaines difficultés rencontrées par le rédacteur non natif dans l'organisation de son texte à rédiger. La notion d'organisation textuelle, dans le sens où il emploie, il l'illustre avec l'analogie de W. Chafe (1976) qui a appelé l'organisation textuelle 'packaging' de façon imagée:

The kind of phenomena at issue here, [...] have to do primarily with how the message is sent and only secondarily with the message itself, just as the packaging of toothpaste can affect sales in partial independence of the quality of the toothpaste inside. (Chafe, 1976; cité in Carter-Thomas 2000: 50)

Chafe illustre ainsi la corrélation entre le contenu d'un texte et sa structure d'organisation par analogie avec celle d'un dentifrice et son emballage.

L'étude de Carter-Thomas traite à la fois le volet d'«argumentation» et celui d'« organisation». En procédant à une analyse thématique, Carter-Thomas effectue une analyse de micro-linguistique qui permet d'identifier formellement les thèmes, les rhèmes phrastiques et le réseau de thèmes qui se construit. Il relève ensuite en quoi consistent les réalisations linguistiques de cette organisation thématique. Cette étape correspond à l'axe premier d'«argumentation» de la rhétorique. Ce n'est que par la suite que l'on procède à l'axe second, celui de l'articulation entre l'organisation thématique et le plan structurel du texte. On peut constater que le passage d'un axe à l'autre est toujours graduel, sans avoir une frontière nette séparant les deux axes connexes dans les pratiques d'analyse, comme nous essayons également de montrer par des carrés colorés superposés dans le schéma 3.

Dans une telle approche la phrase est analysée comme une structure porteuse d'information au sein d'une certaine dynamique textuelle. L'étude de la construction textuelle, par le biais d'une analyse de sa structuration thématique, permet de comprendre comment les rédacteurs maîtrisent le flux d'information et arrivent ainsi à guider l'interprétation du récepteur. (Carter-Thomas, 2000)

Tout type de discours écrit possède des caractéristiques d'organisation discursive distinctes. Des contraintes rhétoriques spécifiques à un genre ou à un autre 
impliquent une structure rhétorique du texte, plus ou moins rigoureuse selon les conventions génériques observées. Chaque genre textuel possède une structure discursive interne qui organise les informations afin de créer un ensemble cohérent. «Un article de recherche scientifique, tout comme un conte de fées par exemple, sera rédigé selon une certaine norme, qui influencera à la fois la présentation et l'organisation de l'information» (Carter-Thomas, 2000). D'ailleurs, le plan rhétorique du texte est aussi influencé par les normes, par là on entend les conventions génériques imposées à un genre textuel dans une communauté partageant la même culture rhétorique que l'on va développer ensuite dans le troisième axe.

\subsection{L'axe du style}

Par «style», on entend les règles ou les conventions d'écriture qui sont stabilisées au sein d'une communauté partageant la même culture rhétorique. Malgré leur caractère quasi institutionnel, ces règles ou conventions restent difficiles à saisir. D'autant qu'elles sont rarement formulées explicitement dans un manuel de rédaction ou moins encore dans un manuel de langue. Cet axe d'observation consiste donc à expliciter les préférences culturelles et les attentes du lecteur à l'égard de divers phénomènes du texte. Bi (2016) a effectué une étude qui compare les normes de la dissertation, un genre scolaire par excellence, en contexte français et en contexte chinois. Ses observations sont portées sur des facteurs culturels, sociaux et éducatifs lesquels constituent le contexte d'écriture.

Elle remarque que «nombreux sont les problèmes concernant les conventions d'écriture qui diffèrent dans les deux cultures éducatives » (Bi, 2016). Elle rejoint l'idée que chaque langue a des préférences claires, de sorte que dans le même genre d'écrit, ce qui est valorisé, toléré ou condamné comme procédés d'écriture, diffère selon la norme de chaque culture. Parmi ces procédés, on trouve les manières d'introduire un sujet, de conclure sur un sujet, de disposer les parties d'un texte, d'aborder le sujet posé, d'élargir à partir du sujet posé, etc. Voici quelques exemples donnés par Bi pour montrer que les degrés d'acception peuvent être extrêmement variés en fonction de la norme rhétorique qui sous-tend une culture éducative et sociale particulière.

Les réactions contrastantes produites chez des lecteurs de cultures différentes montrent bien que certaines spécificités textuelles peuvent paraître étranges aux yeux des lecteurs provenant de culture éducative différente. Cette étrangeté entraîne, de ce fait, des problèmes de communication interculturels. Enfin, elle souligne la prégnance d'un schéma textuel d'origine chinoise, le Qi-Cheng-Zhuan-He, ce qui a permis d'expliquer «les deux spécificités textuelles: l'absence d'explication 
des exemples, l'importance de l'êthos et du pathos à travers les formes d'adresse du discours et, pour terminer, les visées moralisatrices des écrits» (Bi, 2016).

\begin{tabular}{|l|l|l|}
\hline \multirow{2}{*}{ Phénomènes textuels } & \multicolumn{2}{c|}{ Réception du lecteur } \\
\cline { 2 - 3 } & Contexte français & \multicolumn{2}{c|}{ Contexte chinois } \\
\hline schéma question-réponse & valorisant & acceptable \\
\hline schéma réponse-explications & condamnable & acceptable \\
\hline disposer des parties déséquilibrées & condamnable & $\begin{array}{l}\text { acceptable ou valorisant comme } \\
\text { effet de style }\end{array}$ \\
\hline $\begin{array}{l}\text { donner d'emblée une réponse à la question } \\
\text { posée }\end{array}$ & condamnable & $\begin{array}{l}\text { acceptable ou valorisant comme } \\
\text { effet de style }\end{array}$ \\
\hline ne pas annoncer de plan & condamnable & acceptable \\
\hline la juxtaposition des idées sans lien explicite & condamnable & acceptable \\
\hline $\begin{array}{l}\text { la convocation d'exemples donnés sans } \\
\text { explication }\end{array}$ & condamnable & acceptable \\
\hline une mise en retrait de l'auteur & valorisant & acceptable \\
\hline $\begin{array}{l}\text { une manière objective de présenter les } \\
\text { arguments }\end{array}$ & valorisant & acceptable \\
\hline présenter arguments et contre-arguments & valorisant & acceptable \\
\hline anecdotes personnelles & condamnable & acceptable \\
\hline commentaires émotionnels & condamnable & acceptable \\
\hline interactions avec les lecteurs & condamnable & acceptable \\
\hline
\end{tabular}

Les études autour de cet axe défendent l'idée que la rhétorique est un phénomène essentiellement culturel, que l'organisation textuelle est influencée par les spécificités culturelles relevant du contexte propre à la société où la production s'est effectuée.

\section{CONCLUSION}

Les enseignants de L2 évaluent la qualité des textes des apprenants en se fiant à la grammaire et au lexique de surface faute de méthodes d'analyse de l'organisation globale du texte. Et ce toutefois une appréciation de l'organisation d'un texte fera automatiquement partie de tout jugement porté sur la qualité textuelle. En se focalisant seulement sur le niveau micro-linguistique, les enseignants ne fournissent pas aux apprenants tous les outils nécessaires à l'amélioration de la qualité de leurs écrits. En situation d'interculturalité, ce que les auteures transfèrent, malgré tout effort fourni, s'étend de la structure syntaxique, du concept lexico-sémantique, de l'organisation textuelle à l'ensemble des expériences langagière et cognitive intériorisé par un individu scripteur de L2. 
Nous exposons ces trois angles comme les axes constituants des travaux menés sous l'étiquette large de la rhétorique contrastive afin de différencier et mettre en cohérence de divers éléments et phénomènes discursifs décrits et analysés dans les travaux antérieurs. Cette démarche était importante pour tenir compte des résultats issus des recherches qui ont chacune leurs propres objets d'étude rhétorique et terminologie du domaine. Au vu de l'immensité des champs d'étude que recouvre la rhétorique, une systématisation des éléments et des phénomènes traités est indispensable pour mieux situer des recherches déjà effectuées et à venir dans un cadre systémique.

\section{RÉFÉRENCES BIBLIOGRAPHIQUES}

Adam, J.-M. (1985). Le texte narratif. Paris: Nathan.

Adam, J.-M. (2005). La linguistique textuelle: Introduction à l'analyse textuelle des discours. Paris: Armand Colin.

Ali Bouacha, M. (1993). Enonciation, argumentation et discours: le cas de la généralisation. Semen, $8,43-61$.

Anscombre, J.-C. (1983). L'argumentation dans la langue / Jean-Claude Anscombre; Oswald Ducrot. Bruxelles: Mardaga.

Anscombre, J.-C., Ducrot, O. (1984). L'argumentation dans la langue. Revue Philosophique de la France et de l'Etranger, 174, 111-112.

Anscombre, J.-C. (1995). Théorie des topoï. Argumentation, sciences du langage. Paris: Éd. Kimé.

Benveniste, E. (1966). Problèmes de linguistique générale I. Paris: Gallimard.

Benveniste, E. (1974). Problèmes de linguistique générale II. Paris: Gallimard.

BI, X. (2016). Rhétorique de la dissertation: Étude contrastive des conventions d'écriture académique en français et en chinois. Thèse de doctorat - Sorbonne Paris Cité.

Carter-Thomas, S. (2000). La cohérence textuelle pour une nouvelle pédagogie de l'écrit. Paris: L'Harmattan.

Charolles, M. (1978). Introduction au problème de la cohérence des textes. Langue Française, 38, $7-41$.

Chafe, W.L. (1976). Givenness, contrastiveness, definiteness, subjects, topics and points of view. In: C.N. Li (ed.), Subject and Topic (pp. 25-55). New York: Academic Press.

Charolles, M. (1982). Études sur la cohérence et l'interprétation des discours. Thèse d'Etat, l'Université de Franche-Comté.

Charolles, M. (1988). Les études sur la cohérence, la cohésion et la connexité textuelles depuis la fin des années 1960. Modèles Linguistiques, 10(2), 45-66.

Charolles, M. (1988). Les plans d'organisation textuelle: périodes, chaînes, portées et séquence. Pratiques, 57, 3-14.

Combettes, B. (1983). Pour une grammaire textuelle. La progression thématique. Paris: De BoeckDuculot.

Combettes, B. (1988). Pour une grammaire textuelle. La progression thématique. Paris: De BoeckDuculot.

Connor, U., Kaplan, R.B. (eds.). (1987). Writing across languages : Analysis of L2 texts. Reading. MA: Addison-Wesley. 
Connor, U. (1996). Contrastive Rhetoric: Cross-cultural Aspects of Second Language Writing. Cambridge: Cambridge University Press.,

Grabe, W., Kaplan, R.B. (1989). Writing in a Second Language: Contrastive Rhetoric. In : M. Donna, D. Johnson, H. Roen, J. Stephen, Richness in Writing: Empowering ESL Students (pp. 263-283). New York: Longman.

Halliday, M.A.K., Hasan, R. (1976). Cohesion in English. London: Longman.

Hoey, M. (1991). Patterns of Lexis in Text. Oxford: Oxford University Press.

Kaplan, R.B. (1966). Cultural Thought Patterns in Inter-Cultural Education. Language Learning, 16(1-2), Ann Arbor, 1-20.

Kaplan, R.B., Grabe, W. (2002). A modern history of written discourse analysis. Journal of Second Language Writing, 11 (janvier), 191-223.

Purves, A. (1986). Rhetorical communities, the international student and basic writing. Journal of Basic Writing, 5, 38-51.

Purves, A. (1988). Introduction. In: A. Purves (ed.), Writing across languages and cultures (pp. 9-21). Newbury Park, CA: Sage.

Purves, A. (1992). Reflections on research and assessment in written composition. Research in the Teaching of English, 26(1), 108-122.

Saneikashani, N. (2012). Autour des relations conjonctives interphrastiques dans les productions écrites en français langue seconde d'apprenants universitaire: essai d'analyse textuelle. Thèse de doctorat, sous la direction de B. M. Kashema Laurent Masegeta, Université de Strasbourg.

Tirrkonen-Condit, S. (1985). Argumentative Text Structure and Translation. Ph. D. Dissertation. University of Jyvaskyla, Jyvaskyla.

Van Dijk, T.A. (1980). Macrostructures. Hillsdale, NJ : Erlbaum.

Van Dijk, T.A. (1985). Semantic Discourse Analysis. In: T.A. Van Dijk (ed.), Handbook of Discourse Analysis, I : Disciplines of Discourse; II : Dimensions of Discourse; III: Discourse and Dialogue; IV: Discourse Analysis in Society (pp. 103-112). London: Academic.

\section{RÉSUMÉ}

On arrive sans contrainte particulière aujourd'hui à admettre que les actes langagiers étant des objets de traitement de la linguistique sont profondément marqués par le culturel, comme en témoignent également nombre de travaux s'inspirant de la notion du stéréotype, de la politesse, etc., allant de l'échelle phrastique à l'échelle séquentielle discursive ou encore des matières verbales aux matières non verbales. Ce faisant, la linguistique contrastive ouvre la possibilité d'éclairer les similitudes et les différences dans la réalisation des actes de langage des sujets actants ayant des appartenances culturelles diverses.

Dans le domaine de l'enseignement des langues étrangères, en particulier, la perspective contrastive a élargi des modèles possibles d'analyses qui permettent mieux d'appréhender les fonctionnements des langues comparées et les comportements langagiers des locuteurs ayant des langues maternelles différentes.

Ce travail propose un état des lieux des travaux relatifs à la rhétorique contrastive qui ont traité essentiellement des écrits des scripteurs apprenants de langue étrangère et des scripteurs natifs ayant pour but de mettre en lumière des écarts de 'style, argumentation et organisation' (Purves, 1986) qui servent à caractériser des textes provenant des scripteurs des cultures discursives différentes.

Nous posons ces trois angles comme les axes constituants des travaux menés sous l'étiquette large de la rhétorique contrastive afin de différencier et mettre en cohérence de divers éléments et phénomènes discursifs décrits et analysés auparavant. Nous considérons que cette démarche est 
d'autant plus cruciale pour tenir compte des résultats issus des recherches intitulées à multiples manières qui ont chacune leurs propres objets d'étude rhétorique : par exemple, les connecteurs, le procédé de raisonnement déductif/inductif, le style, la convention d'écriture, l'organisation textuelle, la question de la cohésion/cohérence, la structure argumentative, etc.

Mots clés: rhétorique, perspective contrastive, didactique de l'écrit, enseignement de L2.

\begin{abstract}
We can now admit without theoretical constraint that speech acts, being the subject of linguistic studies, are profoundly marked by culture, as proved by many works inspired by the notion of stereotype, politeness, etc., ranging from the sentence pattern to the discursive sequential scale or from verbal materials to non-verbal materials. In doing so, contrastive linguistics opens the possibility of enlightening the similarities and differences in the realization of the speech acts of the sender with different cultural affiliations.

In the field of foreign language teaching, in particular, the contrastive perspective has expanded possible models of analysis that allow better understanding of functions of compared languages and the language writing behaviors of speakers from different cultures.

This work proposes an inventory of the works relating to the contrastive rhetoric, which have mainly dealt with the writings of the learners of foreign language and the native writers with the aim of highlighting the differences in the "style, argumentation and organisation" (Purves, 1986) that characterize texts produced by the writers of different discursive cultures.

We set these three angles as the main research issues of contrastive rhetoric whose aim is to differentiate and to bring into consistency various discursive elements and phenomena described and analyzed so far. We consider that this approach is all the more crucial in order to take account of the results of research entitled in various ways, each of which has its own rhetorical study subjects: for example, connectors; reasoning method, the style, the writing convention, the textual organization, the question of cohesion/coherence, the argumentative structure, etc.
\end{abstract}

Keywords: rhetoric, contrastive perspective, second language writing

\title{
ABSTRAKT
}

Powszechnie się przyjmuje, że akty mowy, będące przedmiotem badań językoznawczych, są wyraźnie nacechowane kulturowo, o czym świadczy szereg prac inspirowanych pojęciem stereotypu, grzeczności itp., a także poświęconych różnorodnym zagadnieniom: od wzoru zdania do dyskursywnej skali sekwencyjnej lub od materiałów werbalnych po materiały niewerbalne. $\mathrm{W}$ ten sposób językoznawstwo kontrastywne otwiera możliwość wyjaśnienia podobieństw i różnic w realizacji aktów mowy dokonywanych przez nadawców wywodzących się z różnych kultur.

W dziedzinie nauczania języków obcych kontrastywna perspektywa badawcza umożliwiła rozszerzenie modeli analizy, które dzięki temu pozwalają lepiej zrozumieć porównywane języki i pisemne zachowania językowe nadawców wywodzących się z różnych kultur.

Niniejszy artykuł proponuje klasyfikację dzieł odnoszących się do retoryki kontrastywnej, które dotyczą głównie tekstów pisanych osób uczących się języka obcego oraz rodzimych pisarzy, w celu podkreślenia różnic pomiędzy „stylem, argumentacją i organizacją” (Purves, 1986), charakteryzujących teksty pisane przez autorów różnych kultur. Te trzy aspekty uznajemy za główne zagadnienia badawcze retoryki kontrastywnej, mającej na celu uporządkowanie różnych elementów 
i zjawisk dyskursywnych dotychczas opisywanych i analizowanych. Uważamy, że takie podejście pozwoli uwzględnić wyniki badań rozmaicie zatytułowanych i obierających sobie za przedmiot badawczy różne zjawiska retoryczne, jak np.: łączniki, metody rozumowania, organizacja tekstowa, struktura argumentacyjna, styl, konwencja wypowiedzi, spójność tekstu (kohezja/koherencja) itp.

Słowa kluczowe: retoryka, perspektywa kontrastywna, teksty pisane 
